\title{
Vacunación en neumococo. Actuación en la farmacia comunitaria
}

\author{
Ricard Casas Jansá', Jesús Molina París², José Antonio Quintano Jiménez³ \\ José Miguel Rodríguez González-Moro ${ }^{4}$ \\ 1. Sociedad Española de Farmacia Familiar y Comunitaria (SEFAC). 2. Sociedad Española de Medicina Familiar y Comunitaria (semFYC). \\ 3. Sociedad Española de Médicos de Atención Primaria (SEMERGEN). 4. Sociedad Española de Neumología y Cirugía Torácica (SEPAR).
}

\section{PALABRAS CLAVE}

Infección por neumococo, vacunación, grupos de riesgo, farmacia comunitaria

\author{
ABREVIATURAS \\ AD0: antidiabéticos orales \\ CCAA: Comunidades Autónomas \\ ECV: enfermedad cardiovascular \\ ENI: enfermedad neumocócica \\ invasiva. \\ EPOC: enfermedad pulmonar \\ obstructiva crónica \\ FLCR: fístula líquido cefalorraquídeo \\ FRCV: factores de riesgo cardiovascular \\ GRADE: Grading of Recommendations \\ Assessment, Development and \\ Evaluation \\ OMS: Organización Mundial de la \\ Salud \\ UBE: unidad de bebida estándar \\ UCI: unidad de cuidados intensivos \\ VIH: virus de la inmunodeficiencia \\ humana \\ VNC13: vacuna conjugada 13 valente \\ VNP23: vacuna polisacárida 23 valente
}

\section{KEYWORDS}

Pneumococcal infection, vaccination, groups at risk, community pharmacy

\section{RESUMEN}

En la elaboración del documento han participado SEFAC, semFYC, SEMERGEN y SEPAR. El objetivo principal era definir los perfiles de paciente susceptibles prioritariamente de la vacunación frente al neumococo considerando los grupos de riesgo, las patologías concomitantes, las posibles consecuencias de una infección neumocócica y la elaboración, a su vez, de un algoritmo de vacunación en el adulto. Se han definido igualmente las indicaciones de la vacuna así como la propuesta de entrevista por parte de la farmacia comunitaria.

El documento, avalado por las principales sociedades científicas médico-farmacéuticas, será de gran utilidad para el farmacéutico comunitario de cara a abordar a pacientes con mayor factor de riesgo de contraer enfermedad neumocócica. Será también una manera muy gráfica para conseguir detectar, asesorar y, llegado el caso, derivar al facultativo médico a todo este tipo de paciente.

\section{Pneumococcal vaccination. Community pharmacy procedure}

SEFAC, semFYC, SEMERGEN and SEPAR were involved in the production of the document. The primary objective was to define the patient profiles most susceptible to be vaccinated against pneumococcus, considering the risk groups, the associated pathologies and the possible consequences of a pneumococcal infection and in turn, to develop a vaccination program in adults. The vaccine indications were also defined, as well as the proposed consultation by the community pharmacy.

The document, backed by the main medico-pharmaceutical science companies, will be very useful to community pharmacies with regards to handling patients with a greater degree of risk of contracting pneumococcal disease. This will also be a very explicit way of detecting, assessing and, as necessary, diverting medical staff to this type of patients.
Recibido: 10/3/2016

Aceptado: 26/5/2016

Disponible online: 1-6-2016
Financiación: Ninguna.

Conflicto de intereses: Los autores declaran no existir conflicto de intereses en relación con el contenido del presente artículo.

Contribución a la autoría: JMRG-M es autor de la Presentación, JMP del apartado Indicaciones de la vacuna antineumocócica, JAOJ del apartado Algoritmo de vacunación antineumocócica en el adulto y $\mathrm{RCJ}$ el de Entrevista en la farmacia comunitaria.

Cite este artículo como: Casas R, Molina J, Quintano JA, Rodriguez JM. Vacunación en neumococo. Actuación en la farmacia comunitaria. Farmacéuticos Comunitarios. 2016 Jun 30; 8(2):35-47. doi:10.5672/ FC.2173-9218.(2016/Nol8).002.06

Autor para correspondencia: Ricard Casas Jansá (rcasas001@cofb.net).

ISSN 1885-8619 @SEFAC (Sociedad Española de Farmacia Familiar y Comunitaria). Todos los derechos reservados. 


\section{PRESENTACIÓN}

José Miguel Rodríguez González-Moro

Sociedad Española de Neumología y Cirugía Torácica - SEPAR

\section{Introducción}

Las infecciones por neumococo, tanto en el niño como en el adulto, constituyen en la actualidad, incluso en los países desarrollados, una importante causa de morbi-mortalidad. El neumococo es un germen, una bacteria, que puede producir infecciones graves en diversos lugares del organismo (pulmón, oído, senos paranasales, meninges, articulaciones). Existen unas cien variedades (serotipos) de neumococo, de los que solo algunos ocasionan infecciones en los seres humanos. Los más vulnerables son los niños pequeños, por debajo de los 5 años y, en particular, los menores de 2 años, aunque también afecta a personas mayores, sobre todo mayores de 65 años o con enfermedades respiratorias y debilitantes que predisponen a las infecciones por este germen. Cuando hablamos de enfermedad neumocócica debemos saber que se trata de enfermedades prevenibles (vacunación) y tratables (antibióticos), aunque en función de la gravedad de la afectación puede llegar a ser una causa importante de muerte.

En este capítulo conoceremos de forma sencilla qué tipo de bacteria es el neumococo y cuáles son los mecanismos de transmisión entre las personas, qué incidencia tienen las enfermedades ocasionadas por el neumococo y cuáles son estas enfermedades que, pese al nombre "neumococo", pueden afectar a otros órganos distintos al pulmón. Por último, hablaremos del tratamiento antibiótico que resulta eficaz para combatir esta infección.

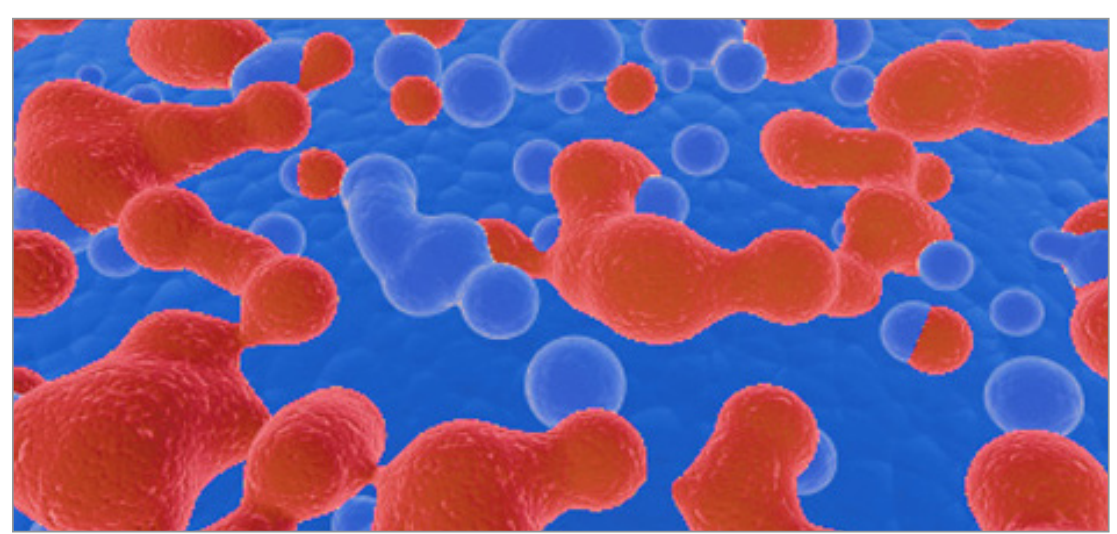

Figura 1 Streptococcus pneumoniae: diplococos gram positivos

\section{¿Qué es el neumococo?}

El neumococo es un tipo de bacteria gram positiva que pertenece a la familia de los estreptococos y forma cadenas cortas o parejas (diplococo), motivo por el que su nombre científico es Streptococcus pneumoniae (figura 1). Esta bacteria se encuentra de manera habitual en la garganta o en las vías respiratorias de los seres humanos. Por lo tanto, pueden existir portadores que no padezcan la enfermedad pero sí la transmitan.

El neumococo se caracteriza por presentar una delgada cápsula de polisacáridos que protege a la bacteria del sistema inmune humano y que le condiciona el serotipo, la virulencia y es la base para el desarrollo de vacunas, como luego veremos en otros capítulos. Los serotipos neumocócicos difieren en las propiedades biológicas, la capacidad patógena e invasora, los síndromes que producen, su capacidad para colonizar la nasofaringe y las resistencias a antibióticos. Así, por ejemplo, los serotipos 1 , 5, 7F, 14 y algunos otros tienen una gran capacidad invasora. Aunque existen más de 90 serotipos distintos y su prevalencia varía regionalmente, solo 11 serotipos son los causantes de más de $2 / 3$ de la enfermedad en el mundo.

\section{¿Cómo se transmite?}

La transmisión del neumococo se hace por vía respiratoria. La bacteria se disemina a través del contacto con personas que están infectadas o con

personas que no están enfermas pero que portan la bacteria en la parte posterior de su nariz. Por esta razón, es muy importante disminuir el número de estos portadores sanos, ya que de esta forma se actúa sobre los mecanismos de contagio. La trasmisión de persona a persona se realiza a partir de la tos y los estornudos, por exposición directa con partículas respiratorias de personas infectadas o que portan la bacteria. La colonización nasofaríngea es la piedra angular de la epidemiología de las infecciones neumocócicas, ya que es el paso previo a la infección, representa el reservorio de la enfermedad y condiciona la selección de resistencias (figura 2). Se ha estimado que entre un 25\% y un 60\% de los niños menores de 4 años están colonizados. En los adultos, la colonización es menos frecuente, pero aumenta en aquellos que conviven con niños. También se ha visto que la utilización de la vacuna conjugada frente al neumococo disminuye la colonización por los serotipos vacunados.

\section{¿Qué infecciones produce?}

El nombre de esta bacteria proviene de su capacidad para producir neumonía, es decir, una infección en el pulmón, sobre todo en niños de menos de 5 años en los que es la primera causa de neumonía bacteriana. Sin embargo, no es la única infección que causa pudiendo ocasionar enfermedades en otras localizaciones del organismo.

De manera esquemática, tal y como se muestra en la figura 3, las infecciones neumocócicas pueden ser invasivas (cuando hay diseminación sanguínea o aislamiento del microorganismo en otros fluidos estériles como el articular, pleural o cefaloraquideo) y no invasivas. Las infecciones no invasivas más comunes son la otitis media, la sinusitis y la neumonía, mientras que enfermedades invasivas, y por tanto más graves, son neumonía bacteriémica, empiema, meningitis, artritis y bacteriemias. S. pneumoniae es una causa frecuente de bacteriemia en pacientes inmunodeprimidos. 


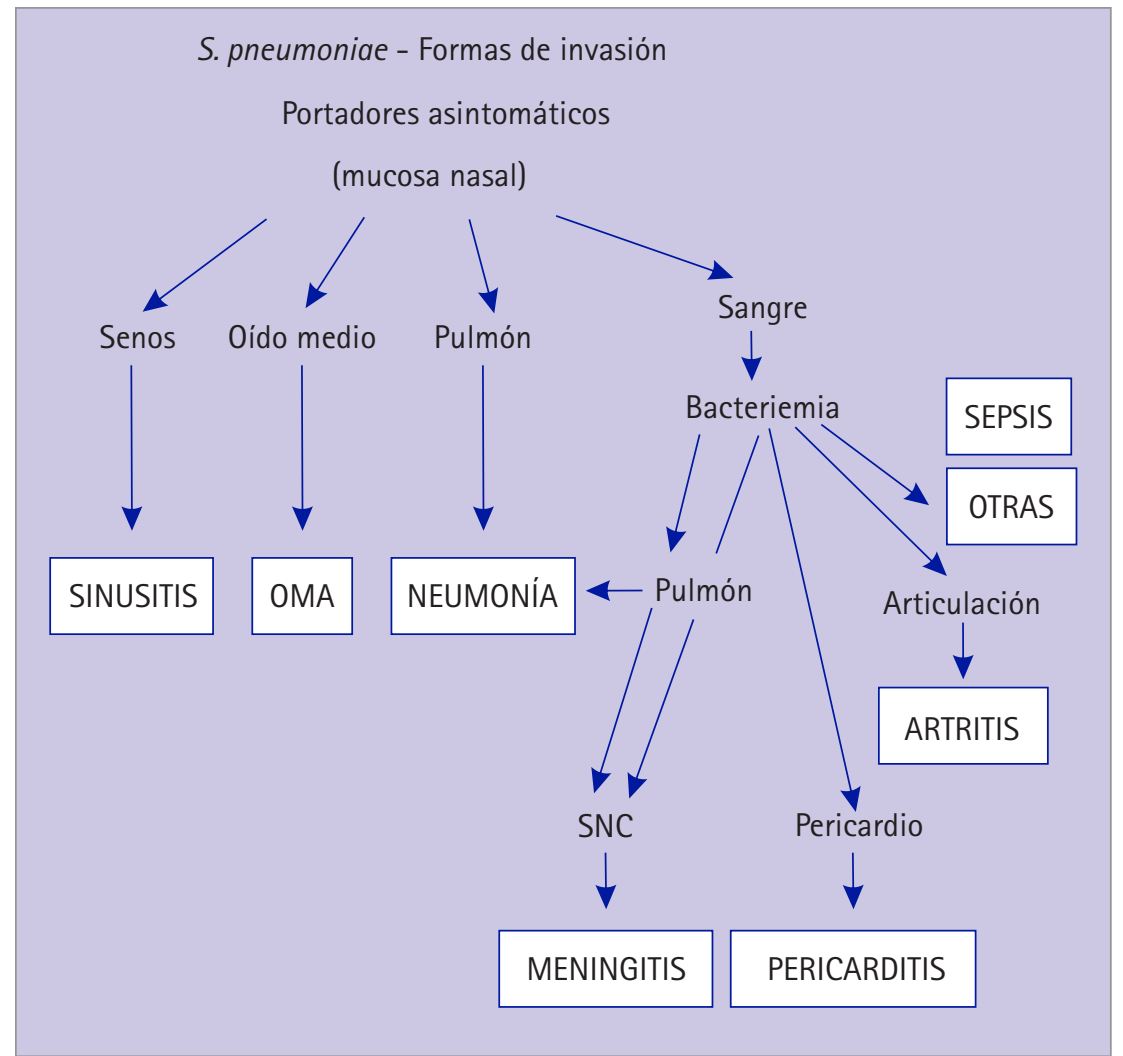

Figura 2 Formas de invasión de los distintos órganos por el neumococo

Las enfermedades producidas por neumococo ocurren todo el año. Sin embargo, las infecciones respiratorias causadas por este germen ocurren con mayor frecuencia durante el invierno y a principios de la primavera.

\section{¿Cuál es la incidencia de infecciones neumocócicas?}

La Organización Mundial de la Salud (OMS) estima que más de 1,1 millones de niños menores de 5 años mueren anualmente en el mundo por neumonía de todas las causas, lo que supondría entre el 10-15\% de todas las muertes infantiles. La tasa de neumonías en España, de todas las etiologías, fue de 420/100.000 habitantes en 1996 y se calcula que el 21,4\% eran de etiología neumocócica. Por otra parte, se considera que el $30-40 \%$ de las otitis medias agudas en nuestro país están producidas por neumococos. Estos datos nos dan una idea aproximada de la importancia de la infección neumocócica en nuestro medio.

En los países industrializados, las infecciones neumocócicas afectan a las edades extremas de la vida. En los niños menores de 2 años, la incidencia de estas infecciones es mayor que en cual- quier otra época de la vida, pero la mortalidad debida a las mismas es relativamente baja y se asocia, casi de forma exclusiva, a meningitis. En los adultos mayores de 65 años, la mortalidad se debe, sobre todo, a neumonía (figura 4).

La incidencia de enfermedad neumocócica invasiva en cualquier población dependerá de su localización geográfica, estación del año, serotipo prevalente, edad y estado de vacuna-

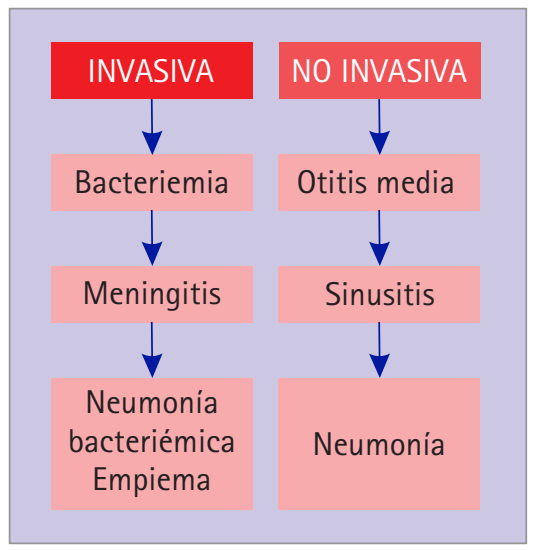

Figura 3 Enfermedades ocasionadas por el neumococo

ción de la población. Aunque puede afectar a cualquier persona, los grupos más vulnerables de tener enfermedad grave son los niños menores de 2 años y los adultos que padecen enfermedades crónicas pulmonares, cardíacas, renales, inmunológicas, diabéticos, fumadores, esplenectomizados, fumadores y los mayores de 65 años.

La mortalidad de la enfermedad neumocócica invasiva puede oscilar del 1\% al 5\% en los pacientes ambulatorios, del 11\% al 44\% en los pacientes hospitalizados y del 34\% al $50 \%$ en aquellos ingresados en una unidad de cuidados intensivos (UCI), especialmente en los que necesitan ventilación asistida. La mortalidad a medio y largo plazo es elevada, con cifras del $8 \%$ a los 90 días, $21 \%$ al año y 36\% a los 5 años.

Si nos referimos a hospitalizaciones por neumonía, el estudio de Gil

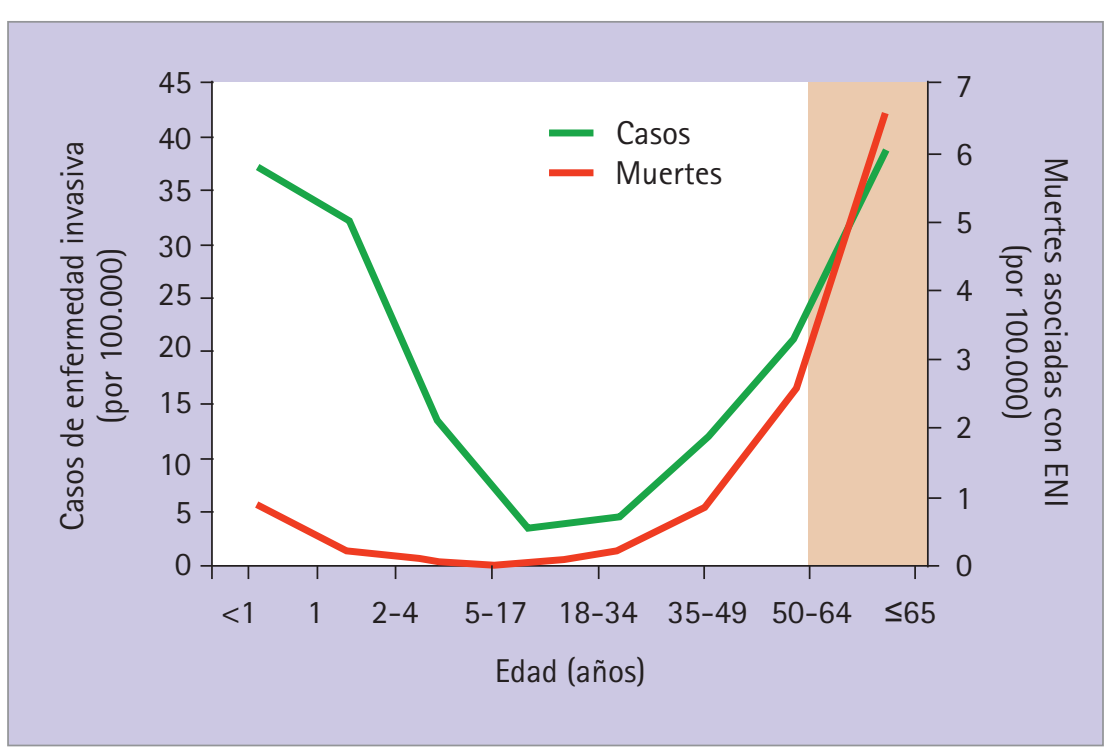

Figura 4 Casos, edades y mortalidad por neumococo 


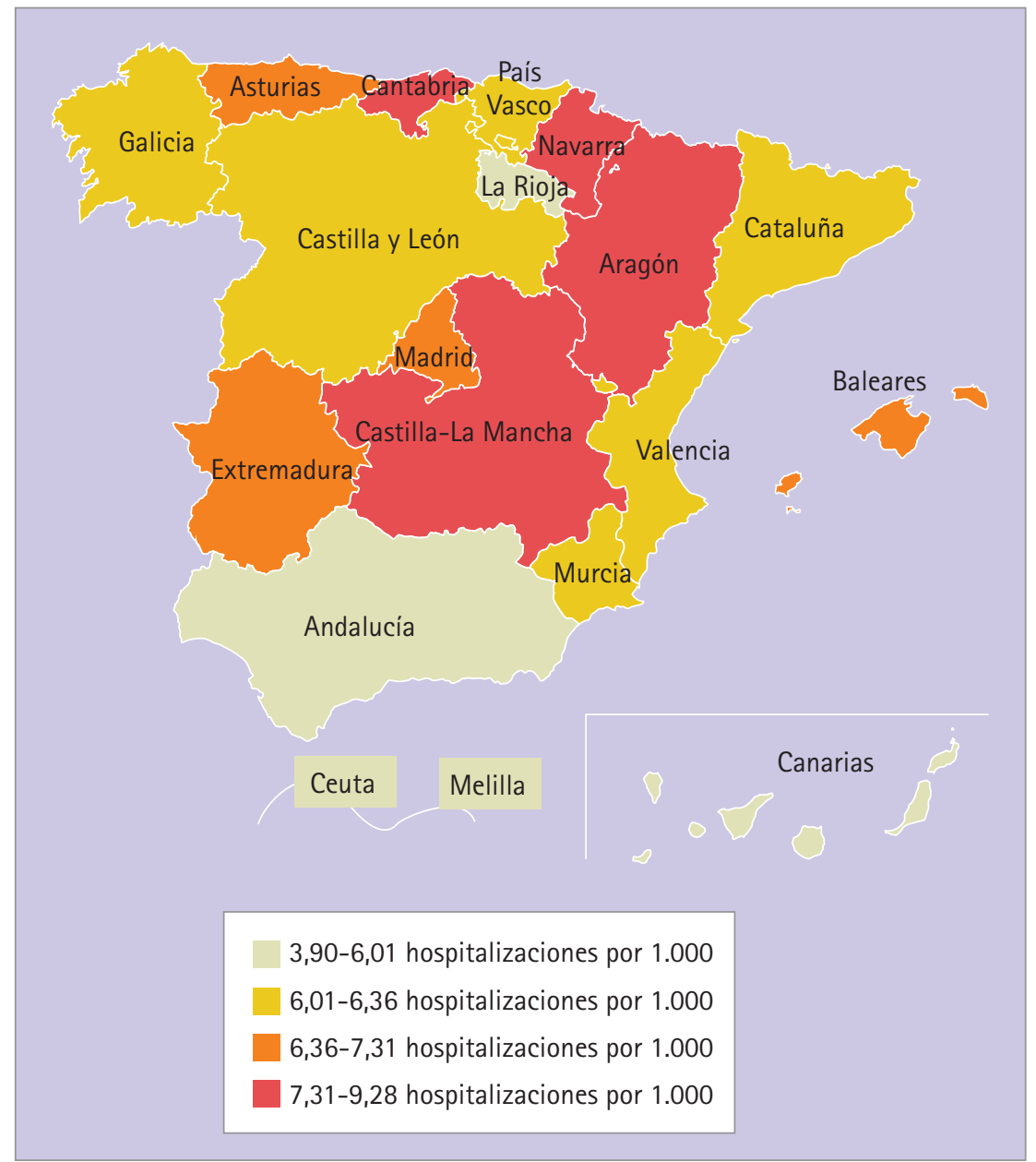

Figura 5 Hospitalizaciones por neumonía en España

Prieto (2011) en España mostró cifras que oscilaron entre los 3,9 casos/1.000 habitantes de comunidades como Andalucía a los 9,28 casos/1.000 habitantes de Castilla-La Mancha, Aragón y Navarra (figura 5).

\section{¿Cuál es el coste económico?}

Según el estudio poblacional de Gil y cols, la neumonía neumocócica durante el periodo de 5 años (20032007) ocasionó 937.884 días de hospitalización en centros públicos españoles. El coste por asistencia hospitalaria por paciente fue de 5.353,80€ debido a todas las causas de neumonías comunitarias y de 4.641,96€ por paciente en el caso de neumonía neumocócica. El coste anual estimado de estas hospitalizaciones fue superior a 479 millones de euros para el conjunto de neumonías comunitarias y de 72,5 factores de riesgo para presentar enfermedad neumocócica.

En segundo lugar, cuando se produce ya una infección hay que utilizar tratamiento con antibióticos, cuyas pautas y dosis dependerá del tipo y gravedad de la afectación. Las infecciones neumocócicas responden a antibióticos beta-lactámicos a dosis elevadas como penicilina o amoxicilina, debiéndose recurrir a cefalosporinas de $3^{a}$ generación como cefotaxima o una quinolona "respiratoria" tipo levofloxacino en los casos de sospecha de resistencias a penicilinas. Cuando se aísla la bacteria hay que determinar su grado de sensibilidad a los diversos antibióticos. Los macrólidos y la clindamicina tienen un papel muy limitado en las infecciones neumocócicas, dadas las tasas de resistencias tan altas que se están encontrando.

\section{Referencias bibliográficas}

1. Centers for Disease Control and Prevention. Pneumococcal disease. In: Atkinson W, Wolfe S, Hamborsky J, eds. Epidemiology and Prevention of Vaccine-Preventable Diseases. 12 ed. Washington, DC: Public Health Foundation; 2011. pp. 233-248.

2. Gil-Prieto R, Garcia-Garcia L, Alvaro-Meca A, Mendez C, Garcia A, de Miguel AG. The burden of hospitalizations for community-acquired pneumonia (CAP) and pneumococcal pneumonia in adults in Spain (2003-2007). Vaccine 2011; 29:412-416. doi:10.1016/j.vaccine.2010.11.025

\section{¿Cuál es el tratamiento?}

En primer lugar, hay que hacer hincapié en la prevención, que se realiza en la actualidad mediante la utilización de vacunas polisacárida y conjugada. Según la OMS, la enfermedad neumocócica es la primera causa de muerte prevenible mediante vacunación en el mundo, no sólo en niños, sino en todas las edades. Por ello, la OMS considera esencial y prioritaria la introducción e implementación de la vacunación frente al neumococo de forma universal en todos los países. El calendario vacunal del niño en España aconseja introducirla de forma sistemática, con distintos grados de financiación pública en las diferentes regiones. En adultos, los diferentes documentos de consenso incluyen la vacunación en personas con
3. Musher DM. Streptococcus pneumoniae. In: Mandell GL, Bennett JE, Dolin R, eds. Mandell, Douglas, Bennets. seases. Philadelphia: Churchill Livingstone Elsevier; 2010. pp. 2623-42.

4. Gutiérrez Rodríguez M, Varela González A, Ordobás Gavín M, Martín Martínez F, García Marín F, Ramos Blázquez B, et al. Invasive pneumococcal disease: Association between serotype, clinical presentation and lethality. Vaccine 2011; 29:5740-5746. doi:10.1016/j.vaccine.2011.05.099

5. Paradiso PR. Pneumococcal conjugate vaccine for adults: a new paradigm. Clin Infect Dis 2012; 55:259-264. doi:10.1093/cid/cis359

6. Up To Date: Pneumococcal vaccination in adults. Daniel M Musher, MD. www. uptodate.com/contents/pneimococcal-vaccination -in-adults 2013. Principles and practice of infectious di- 


\section{INDICACIONES DE LA VACUNA ANTINEUMOCÓCICA}

Jesús Molina París

Sociedad Española de Medicina Familiar y Comunitaria - semFYC

\section{Vacuna polisacárida 23 valente (VNP23)}

Está indicada para la inmunización activa frente a la enfermedad causada por los serotipos de neumococos incluidos en la vacuna. Se recomienda la vacuna en personas de 2 años de edad o mayores que presenten un mayor riesgo de morbilidad y mortalidad por la enfermedad neumocócica. Los grupos específicos de riesgo de personas que se podrían vacunar se determinan sobre la base de las recomendaciones oficiales. La vacuna no es eficaz para la prevención de la otitis media aguda, sinusitis y otras infecciones comunes del tracto respiratorio superior.

En la tabla 1 se recogen las recomendaciones de vacunación con VNP23 en España. Existe bastante homogeneidad entre comunidades autónomas. La principal diferencia se observa con la edad de inicio de la vacunación sistemática, que varía entre 60 y 65 años. El objetivo de la vacunación es la protección de los adultos en los que es más probable contraer la infección neumocócica o en los que la infección puede ser más grave [1]

\section{Vacuna conjugada 13 valente (VNC13)}

Las indicaciones terapéuticas de Prevenar $13^{\circledR}$, vacuna antineumocócica conjugada 13-valente, son [2]:

- Inmunización activa para la prevención de la enfermedad neumocócica invasiva, neumonía y otitis media aguda causadas por Streptococcus pneumoniae desde las 6 semanas hasta los 17 años de edad.

- Inmunización activa para la prevención de la enfermedad neumocócica invasiva y la neumonía causada por Streptococcus pneumoniae en adultos de $\geq 18$ años y personas de edad avanzada.

El uso de Prevenar $13^{\circledR}$ debe ser determinado teniendo en cuenta el riesgo de la enfermedad invasiva y de neumonía en los diferentes grupos de edad, las patologías de base, así como la variabilidad epidemiológica de los serotipos en las diferentes áreas.
Tabla 1 Recomendaciones de vacunación con VNP23 en España

\begin{tabular}{|l}
\hline INMUNODEPRIMIDOS \\
\hline $\begin{array}{l}\text { Inmunodeficiencias humorales o celulares, deficiencias del complemento y trastornos } \\
\text { de la fagocitosis }\end{array}$ \\
\hline Infección por VIH \\
\hline Insuficiencia renal crónica y sindrome nefrótico \\
\hline Leucemia \\
\hline Linfoma \\
\hline Enfermedad de Hodgkin \\
\hline Mieloma múltiple \\
\hline Otras neoplasias \\
\hline Trasplante de órgano sólido \\
\hline Trasplante de progenitores hematopoyéticos \\
\hline Tratamiento inmunosupresor* \\
\hline
\end{tabular}

PERSONAS CON ASPLENIA ANATÓMICA O FUNCIONAL

Anemia de células falciformes

Asplenia adquirida o congénita, disfunción esplénica o esplenectomía

PERSONAS INMUNOCOMPETENTES CON OTRAS PATOLOGÍAS DE BASE

Enfermedad cardiovascular crónica

Enfermedad pulmonar crónica

Diabetes mellitus

Portadores de fístula de LCR

Portadores de implante coclear

Alcoholismo

Hepatopatía crónica o cirrosis hepática

Fumadores $\$$

PERSONAS A PARTIR DE CIERTA EDAD Y/O INSTITUCIONALIZADAS

Personas a partir de 60-65 años

Personas institucionalizadas a partir de 60-65 años

Personas institucionalizadas sin limite de edad

\$Solamente Castilla-La Mancha y Cataluña. ${ }^{*}$ Incluidos quimioterapia, corticoides sistémicos y radioterapia. ${ }^{* *}$ Varía según CCAA. Se recomienda en población general a mayores de 60 años en 5 CCAA (Cantabria, Castilla y León, Cataluña, Madrid y Murcia) y a mayores de 65 en 11 CCAA (Andalucia, Canarias, Castilla La Mancha, C. Valenciana, Extremadura, Galicia, Navarra, Pais Vasco, La Rioja, Ceuta y Melilla). En 3 CCAA (Aragón, Asturias y Baleares) se recomienda a mayores de 65 años institucionalizados. 
En la tabla 2 se esquematizan las recomendaciones de vacunación con VNC13 en algunas comunidades autónomas [1]. Las indicaciones generalmente están restringidas a grupos de riesgo con probabilidad alta de infección neumocócica grave. Debe tenerse en cuenta que algunas condiciones clínicas que no aparecen en las recomendaciones pueden estar con- templadas implícitamente, por ejemplo casos de enfermedad inflamatoria intestinal o tratamientos biológicos que causen distintos tipos de inmunosupresión [1].

En la tabla 3 se pueden ver las instrucciones que están accesibles por comunidades autónomas [3].

Diferentes sociedades científicas realizan también sus propias recomen- daciones. Para la Sociedad Española de Medicina Preventiva, Salud Pública e Higiene [4], las indicaciones médicas en patologías crónicas por ellos consideradas para vacunar con Prevenar $13^{\circledR}$, además de los déficits inmunitarios primarios y secundarios habituales, serían las que pueden verse en la tabla 4 .

En el Consenso sobre la vacunación anti-neumocócica en el adulto

Tabla 2 Recomendaciones de uso de VNC13 en grupos de riesgo por CCAA
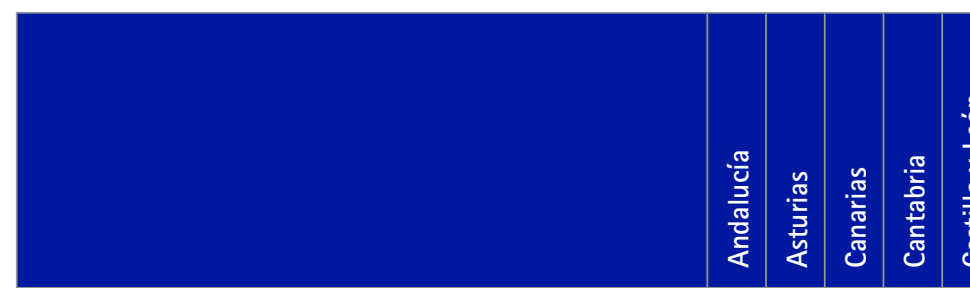

Personas de 50 años o más con factores de riesgo Si

Adultos sin limite de edad con factores de riesgo

Si $\quad$ Si

Si

Inmunodeficiencias humorales o celulares,

deficiencias del complemento y trastornos de la fagocitosis

Leucemia, linfoma, enfermedad de Hodgkin, mieloma múltiple

Insuficiencia renal crónica avanzada

Insuficiencia renal crónica (1)

Sindrome nefrótico

Infección por VIH

Trasplante de órgano sólido

Trasplante de progenitores hematopoyéticos

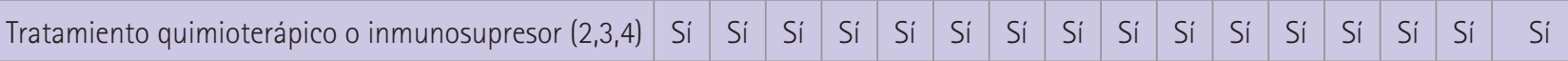

Asplenia (5)

Receptores de concentrados de factores de la coagulación

Fistulas de líquido cefalorraquideo

Portadores de implante coclear

Hepatopatía crónica o cirrosis hepática

Alcoholismo

Antecedentes de ENI (puede requerir confirmación por PCR o cultivo)

Personas $<18$ años incluidas en las recomendaciones

Personas $<18$ años con recomendaciones más amplias

Si Si Si Si $\quad$ Si

Si Si Si Si Si Si

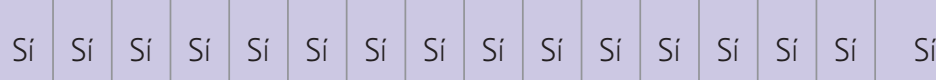

$\begin{array}{lllllllllll}\text { Si } & \text { Si } & \text { Si } & \text { Si } & \text { Si } & \text { Si } & \text { Si } & \text { Si } & \text { Si } & \text { Si }\end{array}$

\begin{tabular}{llll|ll|l|l|l|l|l} 
Si & Si & Si & Si & Si Si & Si & Si & & Si
\end{tabular}

$\begin{array}{llllllllllllllll}\text { Si } & \text { Si } & \text { Si } & \text { Si } & \text { Si } & \text { Si } & \text { Si } & \text { Si } & \text { Si } & \text { Si } & \text { Si } & \text { Si } & \text { Si } & \text { Si } & \text { Si } & \text { Si }\end{array}$

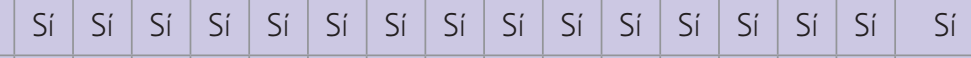

(1) Castilla y León, Madrid, Murcia y País Vasco incluyen explícitamente hemodiálisis. (2) Cataluña y Valencia incluyen explícitamente neoplasias. (3) Cataluña, Murcia y Valencia incluyen explícitamente los corticoides sistémicos a dosis altas y larga duración y la radioterapia. Castilla y León la radioterapia. (4) C. Valenciana incluye de forma explícita anti-TNF, metrotrexate y otros agentes biológicos. (5) Incluye asplenia congénita adquirida, drepanocitosis y esplenectomía. Asturias, Galicia y Murcia incluyen disfunción esplénica por enfermedad celiaca. Resolución de la DG. de Cartera Básica de Servicios del SNA y Farmacia de 12 de julio de 2012 (Valencia septiembre de 2012). 
Tabla 3 Instrucciones por comunidad autónoma [3]

\begin{tabular}{|c|c|c|c|}
\hline & \multirow[t]{2}{*}{ Edad 18-49 } & \multicolumn{2}{|c|}{$>50$ años } \\
\hline & & G. Ministerio & Adicionales \\
\hline Galicia & Igual que mayores de 50 & Sí & $\begin{array}{l}\text { Asplenia. Implante } \\
\text { coclear. FLCR. ENI } \\
\text { previa. Enfermedad } \\
\text { hepática crónica } \\
\text { grave }\end{array}$ \\
\hline País Vasco & & Sí & $\begin{array}{l}\text { Asplenia. Implante } \\
\text { coclear. FLCR. ENI } \\
\text { previa }\end{array}$ \\
\hline Navarra & Igual que mayores de 50 & Sí & Asplenia \\
\hline Cataluña & Igual que mayores de 50 & Sí & $\begin{array}{l}\text { Asplenia. Implante } \\
\text { coclear. FLCR. Otras } \\
\text { inmunodeficiencias }\end{array}$ \\
\hline Valencia & Igual que mayores de 50 & Sí & $\begin{array}{l}\text { Asplenia. Implante } \\
\text { coclear. FLCR }\end{array}$ \\
\hline Murcia & Igual que mayores de 50 & Sí & $\begin{array}{l}\text { Asplenia. Implante } \\
\text { coclear. FLCR. ENI } \\
\text { previa. Hepatopatía } \\
\text { crónica }\end{array}$ \\
\hline Madrid & Igual que mayores de 50 & Sí & $\begin{array}{l}\text { Asplenia. Implante } \\
\text { coclear. FLCR. } \\
\text { Hepatopatía. } \\
\text { Alcoholismo. } \\
\text { Receptores de } \\
\text { factores de } \\
\text { coagulación. ENI } \\
\text { previa }\end{array}$ \\
\hline La Rioja & Igual que mayores de 50 & Sí & $\begin{array}{l}\text { Asplenia. Implante } \\
\text { coclear. FLCR }\end{array}$ \\
\hline Asturias & Igual que mayores de 50 & Sí & No \\
\hline Castilla y León & Igual que mayores de 50 & Sí & $\begin{array}{l}\text { Asplenia. Implante } \\
\text { coclear. FLCR. ENI } \\
\text { previa. Hepatopatía } \\
\text { crónica }\end{array}$ \\
\hline Extremadura & Igual que mayores de 50 & Sí & $\begin{array}{l}\text { Asplenia. Hepatopatía } \\
\text { crónica }\end{array}$ \\
\hline Baleares & Igual que mayores de 50 & Sí & NO \\
\hline
\end{tabular}

FLCR: Fístula líquido cefalorraquídeo. ENI: Enfermedad neumocócica invasiva.

con patología de base [5], realizado por 16 sociedades científicas, se diferencian dos grupos, inmunodeprimidos e inmunocompetentes con patología de base (tabla 5).

Se hace especial hincapié en adultos con patología de base, para los que se realiza una clara recomendación de vacunación. Estos adultos con patología de base deben vacunarse frente a neumococo y recibir, preferentemente, al menos una dosis de VNC 13, que se administrará siempre en primer lugar [5] (tabla 6).
En el documento del Ministerio de Sanidad, Servicios Sociales e Igualdad [1] se establecen recomendaciones de vacunación en grupos de riesgo en la población de 5 y más años de edad (tabla 7), teniendo en cuenta las revisiones recientes llevadas a cabo en varios países afines al nuestro según la evidencia disponible en el momento. En concreto, se han tenido en cuenta las recomendaciones de EEUU (ACIP, 2014), Canadá (Agencia de Salud Pública, 2014), Australia (Department of Health, 2014), Dinamarca
(Statens Serum Institute, 2014), Suiza (Office Fédéral de la Santé Publique, 2014), Reino Unido (Health Protection Agency, 2014) y Francia (Haut Conseil de la Santé Publique, 2013). Se ha dado un mayor peso a las recomendaciones formuladas en función de la calidad de la evidencia, generalmente valorada utilizando la herramienta GRADE (Grading of Recommendations Assessment, Development and Evaluation) o adaptaciones de la misma. Aunque se observa una cierta heterogeneidad, se observa coincidencia en unos grupos específicos como indicación para administrar VNC13 y/o VNP23.

\section{Referencias bibliográficas}

1. Ministerio de Sanidad, Servicios Sociales e Igualdad. [Internet]. Grupo de trabajo vacunación frente a neumococo en grupos de riesgo 2015 de la Ponencia de Programas y Registro de Vacunaciones. Vacunación frente a neumococo en grupos de riesgo. Comisión de Salud Pública del Consejo Interterritorial del Sistema Nacional de Salud. 2015. Disponible en: https:// www.msssi.gob.es/profesionales/saludPublica/prevPromocion/vacunaciones/docs/Neumococo_Gruposriesgo. pdf

2. AEMPS. [Internet]. Ficha Técnica de Prevenar 13. 2014. Disponible en: http://www.ema.europa.eu/docs/ es_ES/document_library/EPAR_-_Product_Information/human/001104/ WC500057247.pdf

3. Consejería de Sanidad Generalidad Valenciana. [Internet]. Relación de medicamentos CPD con sus indicaciones. 2012. Disponible en: http://www.google.com/url?url=http://www2.san.gva. es/cas/prof/dgf/pdf/0ctubre_2012

4. Dominguez V (coordinador), Arrazola P, Campins M, Chamorro J, de Diego J, Fenoll A, et al. [Internet]. Recomendaciones de vacunación antineumocócica en el adulto por indicación médica. 2012. Disponible en: http:// www.sempsph.com/images/stories/ recursos/pdf/protocolos/2012/Recom_Vac_Antineumococica_SEMPSPH.pdf

5. Picazo JJ, González-Romo F, García Rojas A, Pérez-Trallero E, Gil Gregorio P, de la Cámara R, et al. Consenso sobre la vacunación anti-neumocócica en el adulto con patología de base. Rev Esp Quimioter 2013; 26(3):232-252.

6. Almirall J, Bello S, Riesco Miranda JA, Romero Palacios PJ, Jiménez Ruíz CA, Blanquer J, et al. Vacuna neumocócica en fumadores. Prevención del tabaquismo. 2012; 14(1):8-10. 
Tabla 4 Condiciones médicas subyacentes u otras indicaciones [4]

\section{Condiciones médicas subyacentes u otra indicación}

\begin{tabular}{|c|c|}
\hline Enfermedad hepática crónica & Incluye cirrosis, atresia biliar y hepatitis crónica \\
\hline Enfermedad respiratoria crónica (incluye asma) & $\begin{array}{l}\text { Incluye enfermedad pulmonar obstructiva crónica (EPOC), bronquitis } \\
\text { crónica, enfisema, fibrosis quística y asma }\end{array}$ \\
\hline Enfermedades crónicas del corazón & $\begin{array}{l}\text { Incluye cardiopatía isquémica que requirió tratamiento, cardiopatía } \\
\text { congénita, hipertensión con complicaciones cardiacas, insuficiencia } \\
\text { cardiaca crónica y valvulopatías }\end{array}$ \\
\hline Diabetes & $\begin{array}{l}\text { Incluye diabetes mellitus tipo } 1 \text { y } 2 \text {, tratada con insulina o con } \\
\text { hipoglucemiantes orales; excluye la diabetes controlada sólo con la dieta }\end{array}$ \\
\hline Fistulas de líquido cefalorraquídeo & $\begin{array}{l}\text { Incluye individuos con fístulas de líquido cefalorraquídeo con válvulas de } \\
\text { derivación en casos de hidrocefalia (tras traumatismo o cirugía cerebral) }\end{array}$ \\
\hline \multicolumn{2}{|l|}{ Implantes cocleares } \\
\hline Enfermedades hereditarias & Incluye anomalías cromosómicas y síndrome de Down \\
\hline Tabaquismo & $\begin{array}{l}\text { Incluye los fumadores activos de } \geq 20 \text { cigarrillos al día y que no padecen } \\
\text { ninguna condición de comorbilidad mencionadas en la tabla; los } \\
\text { exfumadores de al menos } 20 \text { cigarrillos al día (consumo histórico de } \geq 10 \\
\text { paquetes/año) y un máximo de } 10 \text { años de abandono del hábito [6] }\end{array}$ \\
\hline Alcoholismo crónico & $\begin{array}{l}\text { Incluye los bebedores de riesgo, aquellas personas con consumo superior } \\
\text { al limite de riesgo: } \leq 280 \mathrm{~g} / \mathrm{semana} 0 \leq 28 \mathrm{UBE}^{*} \text { en hombres } \mathrm{y} \leq 168 \mathrm{~g} / \\
\text { semana } 0 \leq 17 \text { UBE en mujeres }\end{array}$ \\
\hline Internos en un centro o residencia de larga estancia & $\begin{array}{l}\text { Incluye a todos los institucionalizados, independientemente de su edad o } \\
\text { afección crónica }\end{array}$ \\
\hline Miscelánea & $\begin{array}{l}\text { Incluye aquellos con antecedente de padecer una ENI, lupus (LES), } \\
\text { tratamiento con fármacos anticonvulsivantes, enfermedad de injerto } \\
\text { contra-huésped y sarcoidosis }\end{array}$ \\
\hline
\end{tabular}

*UBE: unidad de bebida estándar = 10 gramos de alcohol puro. Recomendaciones semFYC Alcohol. semFYC, Barcelona 2000.

Tabla 5 Pacientes considerados inmunodeprimidos o inmunocompetentes con otras patologías de base o factores de riesgo
- Enfermedad de Hodgkin, leucemia, linfoma

- Mieloma múltiple

- Enfermedad renal crónica estadio 4-5

- Enfermedad renal crónica estadio 3 con riesgo aumentado (síndrome nefrótico, diabetes mellitus o tratamiento con inmunosupresores)

- Trasplante de órgano sólido o de células hematopoyéticas

- Tratamiento quimioterápico o inmunosupresor

- Infección por VIH

- Enfermedad reumatológica inflamatoria autoinmune

- Enfermedad inflamatoria intestinal (incluye enfermedad de Crohn y colitis ulcerosa)

- Enfermedad respiratoria crónica (incluye EPOC, asma grave y patología intersticial difusa)

- Enfermedad hepática crónica (incluye cirrosis)

- Enfermedad cardiovascular crónica (incluye cardiopatía coronaria, insuficiencia cardiaca congestiva y accidente cerebrovascular)

- Diabetes mellitus en tratamiento con antidiabéticos orales (ADO) o insulinodependientes

- Tabaquismo

- Abuso del alcohol 
Tabla 6 Recomendaciones de vacunación en el adulto con patología de base

\begin{tabular}{|c|c|c|}
\hline & No vacunados previamente & $\begin{array}{l}\text { Previamente vacunados con VNP23 } \\
\qquad(\geq 1 \text { año) }\end{array}$ \\
\hline $\begin{array}{l}\text { Inmunodeprimidos } \\
\text { Fistulas del LCR } \\
\text { Implantes cocleares } \\
\text { Asplenia anatómica o funcional }\end{array}$ & $\begin{array}{c}\text { VNC13 } 4 \rightarrow \text { VNP23 } \\
\text { (intervalo minimo } 8 \text { semanas) }\end{array}$ & $\begin{array}{l}\text { VNC13 } \\
\text { Revacunación con VNP23 si } \geq 5 \text { años de } \\
\text { la } 1^{\text {a }} \text { dosis }\end{array}$ \\
\hline $\begin{array}{l}\text { Inmunocompetentes con otras } \\
\text { patologias de base }\end{array}$ & VNC13 & VNC13 \\
\hline
\end{tabular}

Tabla 7 Resumen de recomendaciones de vacunación según grupo de riesgo

\begin{tabular}{|c|c|c|}
\hline Grupos de riesgo & Pauta recomendada & Intervalo entre vacunas \\
\hline \multicolumn{3}{|l|}{ INMUNODEPRIMIDOS } \\
\hline $\begin{array}{l}\text { Inmunodeficiencias humorales o celulares, } \\
\text { deficiencias del complemento y trastornos de la } \\
\text { fagocitosis }\end{array}$ & VNC13 + VNP23* & Al menos 8 semanas \\
\hline $\begin{array}{l}\text { Leucemia, linfoma, mieloma múltiple, enfermedad } \\
\text { de Hodgkin }\end{array}$ & VNC13 + VNP23* & Al menos 8 semanas \\
\hline Otras neoplasias & VNC13 + VNP23* & Al menos 8 semanas \\
\hline Infección por VIH & VNC13 + VNP23* & Al menos 8 semanas \\
\hline Insuficiencia renal crónica y sindrome nefrótico & VNC13 + VNP23* & Al menos 8 semanas \\
\hline Tratamiento inmunosupresor $\neq$ & VNC13 + VNP23* & Al menos 8 semanas \\
\hline Trasplante de progenitores hematopoyéticos & 3d VNC13 + VNP23* & 24 meses \\
\hline Trasplante de órgano sólido & VNC13 + VNP23* & Al menos 8 semanas \\
\hline Asplenia anatómica funcional & VNC13 + VNP23* & Al menos 8 semanas \\
\hline \multicolumn{3}{|c|}{ PERSONAS INMUNOCOMPETENTES CON LAS SIGUIENTES PATOLOGIAS } \\
\hline Fistula de líquido cefalorraquídeo & VNC13 + VNP23 & Al menos 8 semanas \\
\hline Portadores de implante coclear & VNC13 + VNP23 & Al menos 8 semanas \\
\hline Antecedentes de ENI confirmada & VNC13 + VNP23 & Al menos 8 semanas \\
\hline Cirrosis hepática & VNC13 + VNP23 & Al menos 8 semanas \\
\hline Enfermedad cardiovascular crónica & VNP23** & \\
\hline Enfermedad pulmonar crónica & VNP23** & \\
\hline Diabetes mellitus & VNP23** & \\
\hline Hepatopatía crónica & VNP23** & \\
\hline Alcoholismo & VNP23** & \\
\hline Personas $\geq 65$ años de edad & VNP23 & \\
\hline
\end{tabular}

*Revacunación con VNP23 al menos 5 años después. \#Incluye tratamientos con esteroides a dosis inmunosupresoras o con agentes biológicos.

**Revacunación con VNP23 a partir de los 65 años, siempre que hayan transcurrido más de 5 años desde la dosis anterior. 


\section{ALGORITMO DE VACUNACIÓN ANTINEUMOCÓCICA EN EL ADULTO} José Antonio Quintano Jiménez

Sociedad Española de Médicos de Atención Primaria - SEMERGEN

La enfermedad neumocócica es la primera causa de muerte prevenible en el mundo en niños y adultos [1]. Incluye la neumonía adquirida en la comunidad y la forma más grave que es la enfermedad neumocócica invasiva (ENI).

Existen unos factores de riesgo y enfermedades subyacentes que aumentan el riesgo de padecer enfermedad neumocócica [2]. El tabaquismo es un factor de riesgo importante en la aparición de enfermedad neumocócica (tabla 8).

El problema de salud pública que supone la enfermedad neumocócica justifica estrategias de prevención como la vacunación de la población en riesgo y la Organización Mundial de la Salud (OMS) considera prioritaria la vacunación frente al neumococo en todos los países [3].

La evidencia científica actual muestra que la inmunización con la vacuna polisacárida 23 valente (VNP23) es coste/efectiva [4] y a la utilización de vacuna conjugada 13 valente (VNC-13) en adultos como una recomendación de categoría A [5].

Mostramos un algoritmo (figura 6) para la vacunación antineumocócica basado en las recomendaciones de diversos consensos multidisciplinarios sobre vacunación antineumocócica en adultos [6-10].

\section{Referencias bibliográficas}

1. Centers for Disease Control and Prevention. Pneumococcal disease. En: Atkinson W, Wolfe S, Hamborsky J,

Tabla 8 Eficacia de la cesación tabáquica en función del tipo de intervención

\begin{tabular}{|l|c|c|c|}
\hline & Sin tratamiento & $\begin{array}{c}\text { Consejo } \\
\text { breve }\end{array}$ & $\begin{array}{r}\text { Tratamiento } \\
\text { conductual }\end{array}$ \\
\hline Sin fármacos & $5 \%$ & $10 \%$ & $15 \%$ \\
\hline Con fármacos & $10 \%$ & $20 \%$ & $30 \%$ \\
\hline
\end{tabular}

editors. Epidemiology and prevention of vaccine-preventable diseases. 12a ed. Washington DC: Public Health Foundation; 2011, 233-48.

2. Chidiac C. Pneumococcal infections and adult with risk factors. Med Mal Infect 2012 ; 42:517-524. doi:10.1016/j. medmal.2012.04.003

3. World Health Organization (WHO). 23-valent pneumococcal polysaccharide vaccine. WHO position paper. Wkly Epidemiol Rec. 2008; 83:37384.

4. Ogilvie I, Khoury AE, Cui Y, Dasbach E, Grabenstein JD, Goetghebeur M. Cost-effectiveness of pneumococcal polysaccharide vaccination in adults: a systematic review of conclusions and assumptions. Vaccine 2009; 27:4891-4904. doi:10.1016/j. vaccine.2009.05.061

5. Tomczyk S, Bennett NM, Stoecker C, Gierke R, Moore MR, Whitney CG, et al. Use of 13-valent pneumococcal conjugate vaccine and 23-valent pneumococcal polysaccharide vaccine among adults aged $\geq 65$ years: recommendations of the Advisory Committee on Immunization Practices (ACIP). MMWR Morb Mortal Wkly Rep 2014; 63:822-825.

6. Picazo JJ, González-Romo F, García Rojas A, Pérez-Trallero E, Gil Gregorio $\mathrm{P}$, de la Cámara $\mathrm{R}$, et al. Consenso sobre la vacunación anti-neumocócica en el adulto con patología de base. Rev Esp Quimioter 2013; 26:232-252.

7. Recomendaciones de vacunación antineumocócica en el adulto por indicación médica. Sociedad Española de Medicina Preventiva, Salud Pública e Higiene. Revista de Medicina Preventiva 2012; XVIII: 1-33. http://www. sempsph.com/images/stories/recursos/pdf/protocolos/2012/Recom_Vac Antineumococica_SEMPSPH.pdf

8. Jiménez Ruiz CA, Solano Reina S, Riesco Miranda JA, Altet Gómez N, Signes-Costa Miñana J, Lorza Blasco $\mathrm{J}$, et al. Recomendaciones para la vacunación neumocócica en fumadores. Prev Tab. 2012; 14:174-177.

9. Casas Maldonado F, Alfageme Michavila I, Bachilón Cohen VS, Peis Redondo JI, Vargas Ortega DA. Recomendación de la vacuna antineumocócica en las enfermedades respiratorias crónicas. Rev Esp Patol Torac. 2014; 26(2) Suplemento 1:120. doi:10.1016/j.semerg.2014.06.012

10. Jiménez Ruiz CA, Buljubasich D, Sansores R, Riesco Miranda JA, Guerreros Benavides A, Luhning S, et al. Documento de consenso SEPAR-ALAT sobre vacunación antineumocócica en fumadores. Arch Bronconeumol. 2015; 51(7):350-354. doi:10.1016/j. arbres.2014.12.003 


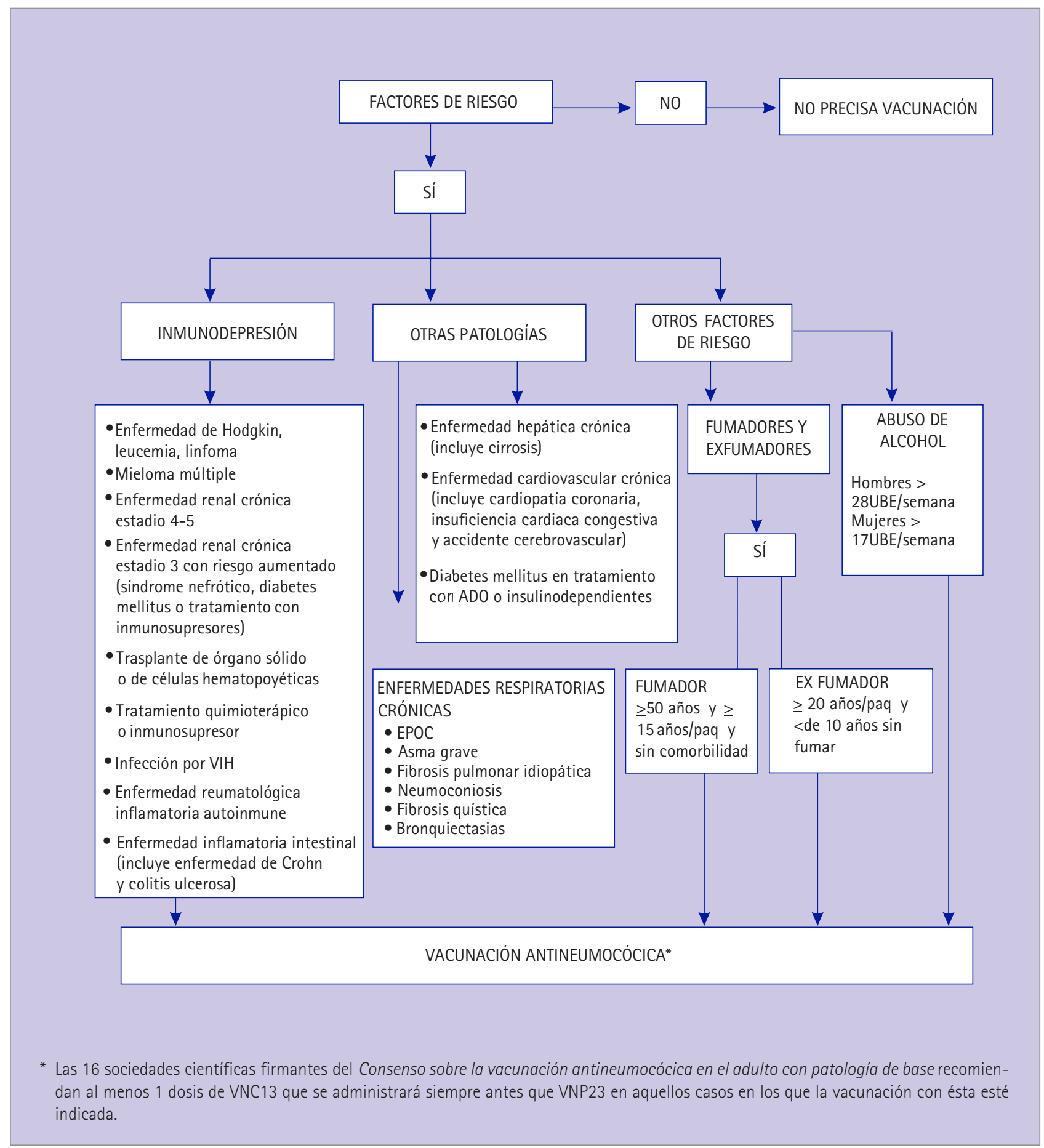

Figura 6 Algoritmo para vacunación antineumocócica en el adulto (basado en Picazo (2013) [6] 


\section{ENTREVISTA EN LA FARMACIA COMUNITARIA}

Ricard Casas Jansá

Sociedad Española de Farmacia Familiar y Comunitaria - SEFAC

Patógeno interviniente: Streptococcus pneumoniae.

La vacunación (cepa muerta/cepa inactiva de neumococo 13 y 23 valente) puede ejercer una prevención en:

- Paciente adulto: enfermedad neumocócica invasiva.

- Paciente joven (6-17 años):

a) Enfermedad neumocócica.

b) Neumonía.

c) Otitis media.

En ambos casos, las citadas enfermedades son producidas por los serotipos vacunales incluidos.

\section{Perfil de paciente}

La enfermedad neumocócica en adultos tiene una tasa de mortalidad notable si existe otra enfermedad de base tal como:

- Enfermedad crónica respiratoria.

- Enfermedad crónica cardiaca.

- Enfermedad crónica renal.

- Enfermedad crónica hepática.

- Diabetes.

En el caso de enfermedad respiratoria existe un mayor riesgo si se trata de EPOC.

En enfermedad cardiaca hay una mayor prevalencia en edad avanzada debido a su vez a una mayor prevalencia de enfermedad cardiovascular (ECV).

La diabetes acarrea una mayor susceptibilidad a infecciones debido a deficiencias en los mecanismos de defensa. Además, suele ir asociada a enfermedades renales y cardiacas.

\section{Entrevista al paciente en la farmacia comunitaria \\ a) Paciente con afectación respiratoria}

- Repasar medicación y asegurarnos que conoce su utilidad y su funcionamiento, sobre todo en caso de inhaladores, básicamente anticolinérgicos, beta-agonistas y corticoides inhalados. A este respecto se puede consultar el programa EPOCA de SEFAC.

- Dispensación.

- Folleto (material de apoyo) sobre la patología respiratoria presente. Iniciamos campaña sanitaria en cuanto a:

1. Repaso del concepto de neumococo y patologías asociadas a riesgo (respiratoria es de las principales).

2. Beneficios de las vacunas antineumocócicas en una sola dosis para adultos.

3. Preguntar al paciente si le queda alguna duda.

\section{b) Paciente diabético}

- Repasar medicación y asegurarnos que conoce su utilidad y su funcionamiento (metformina, sulfonilureas, glitazonas, limitadores de la absorción digestiva y sobre todo las insulinas).

- Dispensación.

- Material de apoyo, folleto diabetes. Iniciamos campaña sanitaria en cuanto a:

1. Repaso del concepto de neumococo y patologías asociadas a riesgo (respiratoria es de las principales).

2. Beneficios de la vacunas antineumocócicas en una sola dosis para adultos.

3. Preguntar al paciente si le queda alguna duda.

c) Paciente con riesgo

cardiovascular

- Repasar medicación, buen uso y conocimiento.

- Asegurarnos de la existencia o no de otros factores de riesgo cardiovascular (FRCV): hipertensión arterial, dislipemia, tabaquismo, obesidad, etc.

- Explicar necesidad de abordar otros FRCV si aún no lo están.

- Ante el tabaquismo, valorar posible carta de derivación a facultativo médico o abordaje en farmacia comunitaria (ver programa CESAR en tabaquismo de SEFAC, disponible en www.campussefac.org).

- Dispensación.

- Material de apoyo, folleto de riesgo cardiovascular. Iniciamos campaña sanitaria en cuanto a:

1. Repaso del concepto de neumococo y patologías asociadas a riesgo (respiratoria es de las principales).

2. Beneficios de la vacuna VCN13 en una sola dosis para adultos.

3. Preguntar al paciente si le queda alguna duda.

En caso de derivación al médico se propone el documento que se presenta en la figura 7 . 
Apreciado Dr/Dra

Su paciente

al que realizamos seguimiento fármaco-terapéutico en esta farmacia y al cual se le han prescrito los siguientes tratamientos:

- Fármaco

- Es fumador

sí

Está en tratamiento/seguimiento de deshabituación

- Es atendido en el día de hoy debido a:

Episodio de

Seguimiento

- Debido a presentar
- Patología

sí NO

Atentamente,

(Firma)

Onfermedad crónica respiratoria

O Enfermedad crónica cardiaca

O Enfermedad crónica renal

Enfermedad crónica hepática

O Diabetes

Le derivamos a su atención de cara a posible propuesta de vacunación frente a neumococo. Como es preceptivo, dejamos tal decisión en sus manos.

Farmacéutico colegiado №.

(Sello Farmacia)

Figura 7 Documento de derivación al médico de familia 